\title{
МЕТОДЫ СВЕРХРАЗРЕШЕНИЯ В ЗАДАЧАХ АНАЛИЗА МЕДИЦИНСКИХ ИЗОБРАЖЕНИЙ
}

\author{
Д. М. Суходолов, А. А. Крыловецкий \\ Воронежский государственный университет
}

Поступила в редакцию 11.03.2018 г.

\begin{abstract}
Аннотация. Исследуется эффективность методов сверхразрешения в задачах анализа медицинских изображений и машинного обучения. Проведен сравнительный анализ результатов поиска особых точек на исходных и увеличенных в два раза методами интерполяции и сверхразрешения изображениях. Рассмотрены различные комбинации современных детекторов и дескрипторов особых точек.

Ключевые слова: детектор, дескриптор, сверхразрешение изображений, интерполяция, обработка изображений, анализ изображений.

Annotation. The effectiveness of methods of superresolution in the problems of analysis of medical images and machine learning is investigated. A comparative analysis of the results of the search for singular points on the original and doubled by the methods of interpolation and superresolution images is carried out. Various combinations of modern detectors and descriptors of singular points are considered.

Keywords: detector, descriptor, superresolution, interpolation, digital image processing, image analysis.
\end{abstract}

\section{ВВЕДЕНИЕ}

В настоящее время создано достаточно много высокотехнологичной медицинской диагностической техники, в том числе и регистрирующей те или иные изображения, доступность ее для врачей и пациентов постоянно растет, тем не менее, доктора не всегда могут извлечь из результатов медицинских исследований достаточно данных для принятия обоснованного решения [1]. Прогресс в области технологий компьютерного зрения позволяет увеличить объем информации, получаемой из медицинских изображений, что поможет врачам в постановке более точных диагнозов, подборе лечения и прогнозировании течения заболеваний.

Актуальным направлением в области применения современных методов для анализа медицинских изображений являются технологии машинного обучения и искусственного интеллекта. Определенные ограничения возникают из-за недостаточности физиче-

() Суходолов Д. М., Крыловецкий А. А., 2018 ского разрешения изображений в реально используемой медицинской технике. Активно развивающиеся в настоящее время методы сверхразрешения позволяют, по нашему мнению, частично снять указанную проблему, повысив как возможности непосредственного визуального анализа, так и увеличив результативность методов машинного обучения при относительно невысоком качестве исходных изображений.

В настоящей работе исследована возможность использования различных методов сверхразрешения медицинских изображений для извлечения большего объема информации и повышения эффективности применения технологий искусственного интеллекта и машинного обучения. С помощью так называемых особых (или ключевых) точек формализуется задача поиска информативных областей на изображениях. Для выделения и описания таких точек используются математические алгоритмы и объекты, называемые детекторами (FAST, Алгоритм Ши-Томаси, Детектор Харриса, SURF, BRISK и MSER [2, 3, $4,5]$ ) и дескрипторами (HOG, BRISK, Block, 
SURF и FREAK $[2,4,5,6])$. Таким образом, в настоящей работе рассмотрено 30 вариантов комбинаций детекторов с дескрипторами.

Для сравнения, кроме методов сверхразрешения (алгоритм ICBI [7], алгоритм Гласнера-Бэгона-Ирани [8] и алгоритм «Улучшения фрактального градиента» [9]), исследована также и интерполяция (бикубическая интерполяция, интерполяция методом ближайшего соседа, билинейная интерполяция) медицинских изображений в контексте возможности получения дополнительной информации. Проведена оценка результаты работы детекторов и дескрипторов на медицинских изображениях при увеличении их разрешения в два раза различными способами.

\section{МЕТОДИКА ЭКСПЕРИМЕНТА}

Для исследования мы использовали набор медицинских изображений, взятых из базы MedPix [10] в количестве $N=100$ штук, содержащих различные патологии: пневмоторакс, аппендицит, цистицеркоз, холецистит, паралич лицевого нерва, грыжу, семиному,

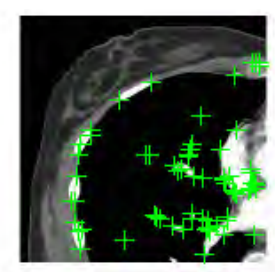

(a)

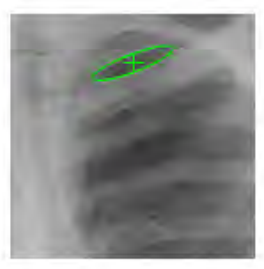

(д)

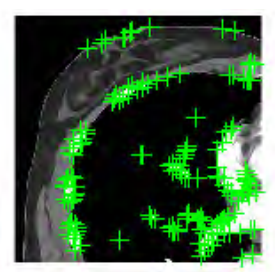

(б)

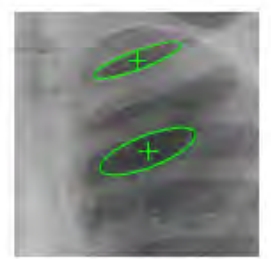

(e)

различные переломы и др. Кроме того, выбрано несколько изображений, представляющих ситуации без патологий. Исходные изображения представлены в форматах JPEG и PNG, размерами от $153 \times 168$ до $615 \times 652$ пикселей в цветовой модели RGB, в целях работы с яркостной составляющей они были трансформированы в цветовую модель YIQ [11].

Для каждого из $\mathrm{N}$ исходных изображений низкого разрешения строятся 6 увеличенных в два раза версий с помощью следующих алгоритмов: бикубическая интерполяция, интерполяция методом ближайшего соседа, билинейная интерполяция, алгоритм ICBI, алгоритм Гласнера-Бэгона-Ирани, алгоритм «Улучшения фрактального градиента». В процессе обработки получено 600 увеличенных изображений в формате $\mathrm{PNG}$, разрешение которых находится в промежутке от $306 \times 336$ до $1230 \times 1304$.

Применяя к каждому изображению комбинацию из детектора и дескриптора, мы получаем набор ключевых точек, характеризующихся соответствующими векторами признаков. Наглядно представим несколько

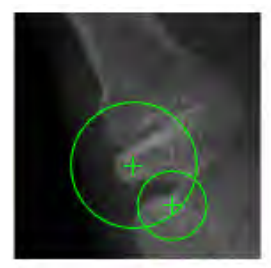

(B)

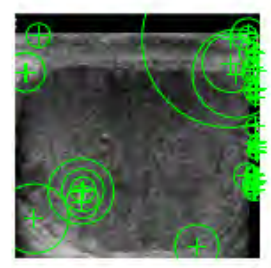

(ж)

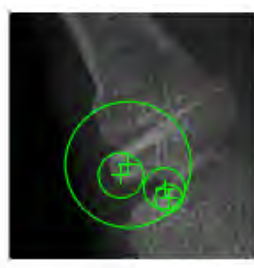

$(r)$

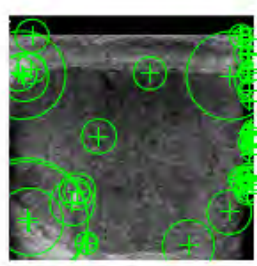

(3)

Рис.1. Визуализачия множества векторов признаков для увеличенных в два раза версий изображений. В скобках указаны детектор, дескриптор и метод увеличения соответственно:

(а) Проникающая кариинома (Harris, HOG, бикубическая интерполячия); (б) Проникающая кариинома (Harris, HOG, Метод Гласнера-Бэгона-Ирани); (в) Перелом (SURF, SURF, билинейная интерполячия); (2) Перелом (SURF, SURF, Улучшение фрактального градиента); (д) Синдром Картагенера (MSER, Freak, метод ближайших соседей); (е) Синдром Картагенера (MSER, Freak, ICBI); (ж) Опухоль клеток Лейдига (BRISK, Block, бикубическая интерполяция);

(3) Опухоль клеток Лейдига (BRISK, Block, Улучшение фрактального градиента) 

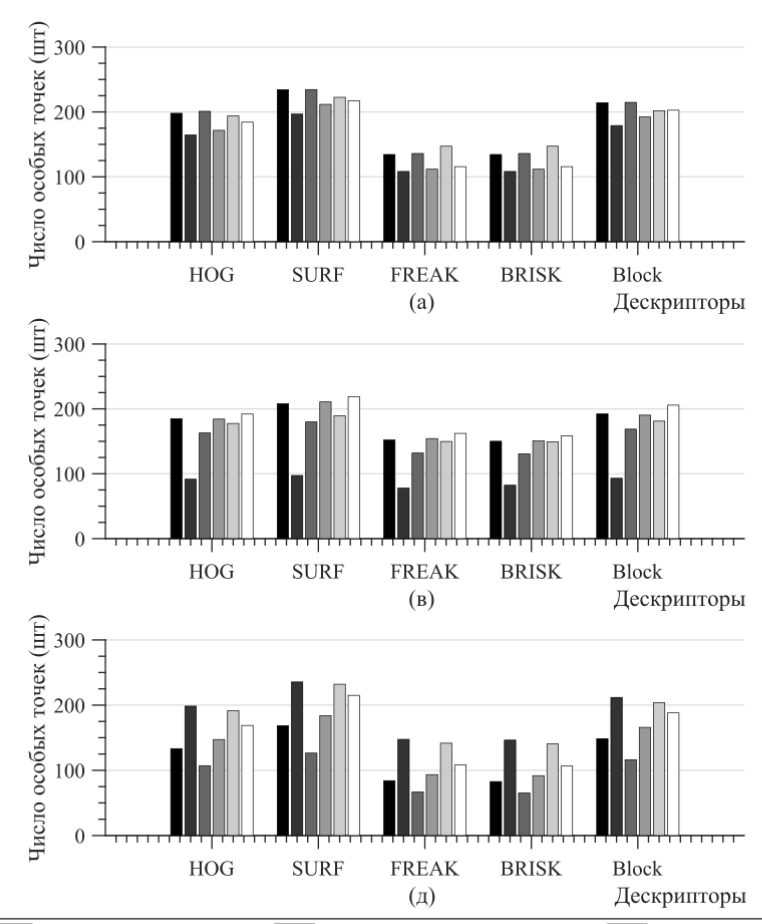
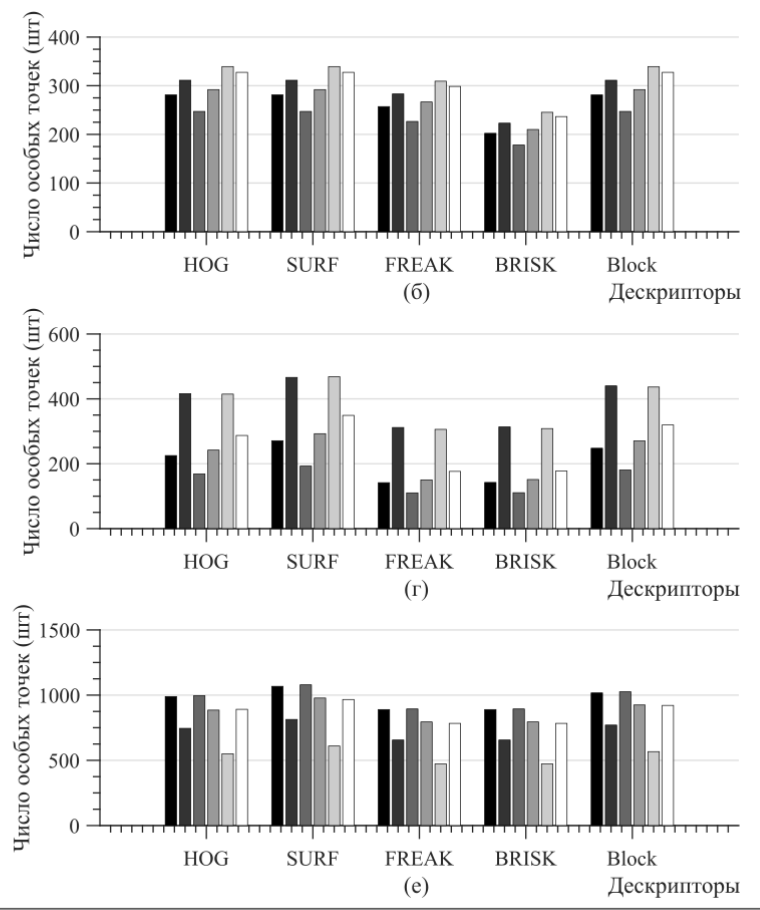

Рис. 2. Визуализачия среднего значения количества особых точек для каждого метода интерполяции для отдельных пар детекторов и дескрипторов: (а) Детектор Харриса, (б) Детектор SURF, (в) Детектор MSER, (г) Детектор FAST, (д) Детектор BRISK, (е) Детектор Ши-Томаси

вариантов улучшения качества детектирования при использовании различных методов повышения разрешения (рис. 1). Всего было использовано 6 детекторов и 5 дескрипторов, в результате получаем 30 их возможных комбинаций. На рис. 2 (а-е) показано среднее значение числа ключевых точек, полученных с помощью детектора и соответствующего дескриптора. Каждый из графиков иллюстрирует применение одного из детекторов. Названия каждой из пяти групп на графике описывают указываемый дескриптор, а в каждой группе, слева направо, представлены разные методы повышения разрешения изображения.

\section{ОБСУЖДЕНИЕ РЕЗУЛЬТАТОВ}

На рис. 2 (а-е) показано усредненное по N изображениям количество найденных особых точек при использовании (a) - детектора Харриса, (б) - детектора SURF, (в) - детектора MSER, (г) - детектора FAST, (д) - детектора BRISK, (е) - детектора Ши-Томаси. Причем на каждом графике на рис. 2 (а-е) слева направо представлены пять различных блоков, соответствующих дескрипторам HOG, SURF, FREAK, BRISK и Block. Каждая из пар, состоящих из детектора и дескриптора, работает с $6 \mathrm{~N}$ входными изображениями, полученными разными способами интерполяции из исходных $\mathrm{N}$ штук низкого разрешения. При использовании детектора Харриса рис. 2(а) наибольшее количество ключевых точек найдено на изображениях, полученных бикубической и билинейной интерполяцией, в паре с дескриптором SURF. Среднее число особых точек для детектора Харриса на рис. 2(a) около 200, усреднение по всем методам повышения разрешения показывает, что методы интерполяции в среднем дают несколько лучший результат (50.46 \% найденных особых точек против 49.54 \%). Для исходных изображений без увеличения в среднем находится порядка 90 особых точек, что значительно меньше.

На рис. 2 (б) приведен график, визуализирующий работу детектора SURF и различных дескрипторов. Можно отметить, что при 
использовании дескрипторов всех видов заметно определенное преимущество методов сверхразрешения Гласнера-Бэгона-Ирани и «Улучшения фрактального градиента». Всего, в среднем, находится порядка 300 особых точек, что лучше, чем в предшествующем случае. Рассматривая среднее значение количества найденных особых точек всеми методами интерполяции и сверхразрешения для данного детектора можно отметить, что 46.67 \% из них найдено методами интерполяции и $53.32 \%$ - методами сверхразрешения. Для текущего набора исходных изображений данным детектором в среднем находится около 100 особых точек.

На рис. 2 (в) представлен график, показывающий результаты работы детектора MSER и различных дескрипторов. В данном случае можно отметить, что при использовании дескрипторов всех видов заметно определенное преимущество метода сверхразрешения «Улучшение фрактального градиента». На увеличенных изображениях в среднем детектор MSER находит порядка 150 особых точек, усреднение по всем методам повышения разрешения дает 44.02 \% ключевых точек на изображениях, увеличенных методами интерполяции и 55.98 \% - методами сверхразрешения. Для исходных изображений найдено в среднем около 65 особых точек.

График, приведенный на рис. 2 (г), показывает результаты поиска ключевых точек детектором FAST, и соответственно, HOG, SURF, FREAK, BRISK и Block дескрипторами. Можно отметить, что при использовании любых дескрипторов заметно преимущество метода сверхразрешения «Улучшения фрактального градиента» и интерполяции методом ближайших соседей. Всего в среднем находится порядка 300 особых точек, хотя для некоторых дескрипторов показатели значительно более высокие. Рассматривая среднее значение количества найденных особых точек, выделяемых на изображения, полученных всеми методами интерполяции и сверхразрешения для данного детектора, можно отметить, что 46.21 $\%$ ключевых точек обнаружено на изображениях, полученных методами интерполяции и 53.79 \% - методами сверхразрешения. В сред- нем около 120 особых точек найдено для изображений в исходном масштабе.

На рис. 2 (д) показаны результаты поиска особых точек с помощью детектора BRISK и различных дескрипторов. Наилучшие результаты имеют метод интерполяции с помощью ближайших соседей и алгоритм Гласнера-Бэгона-Ирани. Всего в среднем находится порядка 200 особых точек, что сходно с результатами для детектора Харриса. Усреднение по изображениям, получаемым различными методами, дает 46.15 \% особых точек на изображениях, полученных методами интерполяции и $53.85 \%$ - методами сверхразрешения. Для исходного набора изображений найдено в среднем приблизительно 60 особых точек.

На рис. 2 (е) график визуализирует работу с детектором на основе алгоритма Ши-Томаси. В данном случае можно отметить, что при использовании дескрипторов всех видов лучшие результаты даёт бикубическая и билинейная интерполяция. Всего в среднем находится порядка 900 особых точек, что является самым высоким показателем из всех детекторов. Для исходных изображений низкого разрешения найдено в среднем около 400 особых точек. Среднее значение количества найденных особых точек на изображениях, увеличенных различными методами, показывает, что $54.01 \%$ ключевых точек найдено на изображениях, полученных методами интерполяции и 45.99 \% методами сверхразрешения.

Из полученных результатов видно, что на увеличенных изображениях получается выделить значительно больше ключевых точек, чем на исходных. Возникает вопрос, не идет ли речь о простом «копировании» особых точек при повышении разрешения изображения. Для ответа на указанный вопрос найден процент несовпадающих точек, выделяемых на увеличенных (табл. 1) и исходных (табл. 2) изображениях. Уникальной считалась точка, в эпсилон окрестности (эпсилон принималось равным 4) по Евклидовой метрике в пиксельных координатах которой не оказывалось других особых точек.

В ходе интерпретации результатов таблицы 1 нетрудно заметить, что среди методов наилучшую дифференциацию точек пока- 
Проиент несовпадающих точек для каждой пары из детектора и дескриптора для увеличенных изображений при эпсилон окрестности равной 4.

По вертикали расположены детекторы, а по горизонтали - дескрипторы

\begin{tabular}{|l|c|c|c|c|c|}
\hline & HOG & SURF & FREAK & BRISK & Block \\
\hline Харрис & 72.97 & 73.01 & 71.79 & 71.78 & 72.60 \\
\hline SURF & 77.51 & 77.51 & 79.24 & 79.19 & 77.51 \\
\hline MSER & 38.92 & 41.18 & 40.48 & 41.01 & 39.72 \\
\hline FAST & 42.89 & 47.41 & 45.66 & 45.65 & 46.48 \\
\hline BRISK & 60.65 & 61.16 & 63.85 & 64.13 & 61.21 \\
\hline Ши-Томаси & 66.88 & 63.75 & 68.79 & 68.79 & 66.14 \\
\hline
\end{tabular}

Таблица 2

Процент несовпадающих точек для каждой пары из детектора и дескриптора для исходных изображений при эпсилон окрестности равной 4.

По вертикали расположены детекторы, а по горизонтали - дескрипторы

\begin{tabular}{|l|c|c|c|c|c|}
\hline & HOG & SURF & FREAK & BRISK & Block \\
\hline Харрис & 69.64 & 66.33 & 66.87 & 66.87 & 68.31 \\
\hline SURF & 75.41 & 75.41 & 76.52 & 76.94 & 75.41 \\
\hline MSER & 47.62 & 49.34 & 43.65 & 50.66 & 48.16 \\
\hline FAST & 46.06 & 42.81 & 48.53 & 47.33 & 41.71 \\
\hline BRISK & 67.49 & 61.80 & 62.77 & 62.97 & 64.52 \\
\hline Ши-Томаси & 63.31 & 61.03 & 64.59 & 64.58 & 62.71 \\
\hline
\end{tabular}

Таблица 3

Количество точек, не обнаруженных при исходном разрешении, но найденных на указанном количестве увеличенных различными рассмотренными методами изображениях

\begin{tabular}{|c|l|l|}
\hline $\begin{array}{c}\text { Точка } \\
\text { выявлена, } \\
\text { раз }\end{array}$ & $\begin{array}{c}\text { Количество точек, усреднённых } \\
\text { по набору исходных } \\
\text { изображений, шт }\end{array}$ & $\begin{array}{c}\text { Количество точек, усреднённых по набору } \\
\text { исходных изображений и количеству пар } \\
\text { детекторов с дескрипторами, шт }\end{array}$ \\
\hline 1 & 8260,88 & 275,36 \\
\hline 2 & 3309,88 & 110,33 \\
\hline 3 & 1371,35 & 45,71 \\
\hline 4 & 509,82 & 16,99 \\
\hline 5 & 243,09 & 8,10 \\
\hline 6 & 22,60 & 0,75 \\
\hline
\end{tabular}

зали пары: SURF и FREAK (79.24 \%), SURF и BRISK (79.19 \%) и другие комбинации с детектором SURF. Для прочих детекторов лучшие результаты в случаях Харрис и SURF (73.01 \%), Харрис и НOG (72.97\%), Харрис и Block (72.60 \%). Также отметим, что наихудшие результаты выявлены для пар MSER и HOG (38.92 \%), MSER и Block (39.72 \%), MSER и $\operatorname{FREAK}(40.48 \%)$.
Сравнивая данные в табл. 2 с данными в табл. 1 можно сделать вывод, что в среднем при повышении разрешения процент несовпадающих точек даже немного вырос.

Для исследования вопроса о том, не являются ли ключевые точки, обнаруживаемые на увеличенных изображениях, случайным шумом, были проведены вычислительные эксперименты, результаты которых представле- 
ны в табл. 3. Найдено число ключевых точек, определяемых сразу несколькими различными детекторами (и дескрипторами).

При этом точки считались одинаковыми, если расстояние между ними не превышало 0.5 пикселя в пиксельных координатах. Из табл. 3 видно, что в среднем $40 \%$ точек находится двумя различными методами, а $17 \%$ точек - тремя методами. При разработке алгоритмов машинного обучения, основываясь на подобных данных, можно присваивать точкам, обнаруживаемым несколькими методами, большие веса как наиболее информативным.

Также был определен процент ключевых точек, обнаруживаемых только одной парой из детектора и дескриптора, и никакой другой. Наибольший результат показала пара из детектора и дескриптора BRISK и SURF $10,97 \%$.

\section{ЗАКЛЮЧЕНИЕ}

Методы сверхразрешения, применяемые к изображениям, в частности медицинским, позволяют увеличить объем извлекаемой из них информации не только при непосредственном наблюдении, но и при использовании алгоритмов машинного обучения.

Исследование результатов поиска ключевых точек на увеличенных различными методами сверхразрешения изображениях показывает некоторое преимущество в работе детекторов MSER и SURF. Максимальную дифференциацию найденных точек показали пары детекторов и дескрипторов SURF и FREAK (79.24 \%), SURF и BRISK (79.19\%).

\section{СПИСОК ЛИТЕРАТУРЫ}

1. Кудрявцев, П. С. Метод повышения качества анализа рентгеновских снимков на основе комбинированных классификаторов / П. С. Кудрявцев, А. А. Кузьмин, О. В. Шаталова, С. А. Филист // Материалы XXIV Всероссийского семинара «Нейроинформатика, её приложения и анализ данных». - 2016. - С. 40-44.
2. Половинкин, П. Н. Детекторы и дескрипторы ключевых точек. Алгоритмы классификации изображений. Задача детектирования объектов на изображениях и методы её решения / П. Н. Половинкин. - 2013. - URL: https://www.hpcc.unn.ru/ file.php?id=760 (дата обращения 23.04.2017).

3. Shi, J. Good Features to Track / J. Shi, C. Tomasi // Proceedings of the IEEE Conference on Computer Vision and Pattern Recognition. 1994. - P. 593-600.

4. Bay, H. SURF: Speeded Up Robust Features / H. Bay, A. Ess, T. Tuytelaars // Computer Vision and Image Understanding. - 2008. V. 110(3). - P. 346-359.

5. Leutenegger, S. BRISK: Binary Robust Invariant Scalable Keypoints / S. Leutenegger, M. Chli, R. Siegwart // Proceedings of the IEEE International Conference on Computer Vision. 2011. - P. 2548-2555.

6. Dalal, N. Histograms of Oriented Gradients for Human Detection / N. Dalal, B. Triggs // IEEE Computer Society Conference on Computer Vision and Pattern Recognition. - 2005. P. 886-893.

7. Giachetti, A. Real time artifact-free image upscaling / A. Giachetti, N. Asuni // IEEE Transactions on Image Processing. - 2011. V. 20(10). - P. 2760-2768.

8. Glasner, D. Super-Resolution from a Single Image / D. Glasner, S. Bagon, M. Irani // IEEE 12th International Conference on Computer Vision. - 2009. - P. 349-356.

9. $X u, H$. Single Image Super-resolution with Detail Enhancement based on Local Fractal Analysis of Gradient / H. Xu, G. Zhai, X. Yang // IEEE Transactions on Circuits and Systems for Video Technology. - 2013. - V. 23(10). - P. 1740-1754.

10. База данных медицинских изображений MedPix. - URL: https://medpix.nlm.nih. gov/ home (дата обращения 21.04.2017).

11. Buchsbaum, W. Color TV Servicing / W. Buchsbaum. - N. J. : Prentice Hall, 1975. $270 \mathrm{p}$. 
Суходолов Д. М. - магистрант кафедры цифровых технологий, факультет компьютерных наук, Воронежский государственный университет.

E-mail: sukhodolov.denis@gmail.com

Крыловецкий А. А. - канд. физ.-мат. наук, доцент кафедры цифровых технологий, факультет компьютерных наук, Воронежский государственный университет.

E-mail: aakryl@cs.vsu.ru
Sukhodolov D. M. - Graduate student of the Department of digital technology, Voronezh State University.

E-mail: sukhodolov.denis@gmail.com

Krylovetsky A. A. - Ph.D. in Physics and Mathematics, Associate Professor of Department of Digital Technologies, Voronezh State University. E-mail: aakryl@cs.vsu.ru 